\title{
Spin paramagnetism in d-wave superconductors
}

\author{
D. Tanasković \\ Institute of Physics, P.O. Box 57, 11080 Belgrade, Yugoslavia \\ Z. Radović \\ Faculty of Physics, University of Belgrade, P.O. Box 368, 11001 Belgrade, Yugoslavia \\ L. Dobrosavljević-Grujić \\ Institute of Physics, P.O. Box 57, 11080 Belgrade, Yugoslavia
}

\begin{abstract}
The Ginzburg-Landau equations are derived from the microscopic theory for clean layered superconductors with $d_{x^{2}-y^{2}}$ pairing symmetry, including the Pauli paramagnetism effect. The upper critical field $H_{c 2}$ parallel to the $c$ axis is calculated. A comparison with the experimental data for YBCO suggests that, relative to the orbital effect, the Pauli paramagnetism contribution to $H_{c 2}$ is significant. The reversible magnetization $M$ in high magnetic fields is also calculated, showing strong temperature dependence of the slope $d M / d H$, as a consequence of the spin paramagnetism. A simple expression for the high temperature spin susceptibility is derived, in a good agreement with the Knight shift measurements on YBCO.
\end{abstract}

PACS: 74.72.-h; 74.25.Ha

\section{INTRODUCTION}

Among the most important properties of the cuprate superconductors are their magnetic properties, reflecting the highly anisotropic layered structure and unfonventional pairing mechanism and symmetry. Nowadays, the dwave pairing symmetry in hole doped cuprates, 2 and quasi-two-dimensional (2D) nature of superconductivity 3 are well established. Although the pairing mechanism is not known, the weak coupling BCS model for 2D d-wave superconductivity, A $_{\text {and }}$ the corresponding Ginzburg-Landau (GL) approximation are remarkably successful.

The existence of superconducting phase in very high magnetic fields and layered structure of high- $T_{c}$ cuprates, makes the paramagnetic effect in the superconducting state much more important than in conventional superconductors 9.0 The purpose of this paper is to derive GL equations for layered d-wave superconductors including the Pauli paramagnetism effect, giving simple analytical expressions for the upper critical field, magnetization, and spin susceptibility, suitable for comparison with experiments.

The GL equations for conventional (isotropic 3D s-wave) superconductors were first derived from BCS theory by Gor'kov 11 For 2P clean superconductors with d-wave pairing GL equations are derived by Ren et al., , and extended by Won and Maki, 7 and Shiraishi et al. 8 to include the higher order derivative terms. However, in the above references the magnetic field influence on the electron spins has been neglected. In their classical papers Maki and Tsuneto studied the effect of the Pauli paramagnetism in conventional superconductors 12,13 Their results mainly refer to the dirty limit.

In many underdoped and overdoped cuprates, where the upper critical fields $H_{c 2}$ and transition temperatures $T_{c}$ are relatively low, the upward, positive curvature in the temperature dependence has been observed, 1455 unlike the Werthamer-Helfand-Hohenberg theory prediction for the conventional superconductors 16 A number of theoretical models for this unusual behavior has been proposed, involving, for example, the influence of the scattering by magnetic impurities and of inhomogeneities,17 the presence of $d_{x y}$ pairing symmetry, 18 and the higher Landau levels effect.19

In the optimally doped cuprates, the temperature dependence of $H_{c 2}$ parallel to the c axis appears to be qualitatively the same as in the conventional superconductors. However, high values of $H_{c 2}$ and the irreversibility effects in optimally doped cuprates pose an obstacle to the study of the field induced transition to the normal state 20 The relevant magnetization measurements of $H_{c 2}$ on high quality single crystals of $\mathrm{YBa}_{2} \mathrm{Cu}_{3} \mathrm{O}_{7-\delta}$ are performed by Welp et al. 21 in a temperature interval of about $8 \mathrm{~K}$ below $T_{c}$. Recently, Nakagawa et al. 22.23 for field parallel and O'Brien et al.24 for field perpendicular to the $c$ axis, reported data from $\mathrm{GHz}$ transport measurements up to $150 \mathrm{~T}$ of $H-T$ phase diagram for $\mathrm{YBa}_{2} \mathrm{Cu}_{3} \mathrm{O}_{7-\delta}$ thin-films in the whole temperature range.

Yang and Sondhi 9 and Won et al. 10 studied theoretically the paramagnetic state of $d_{x^{2}-y^{2}}$ superconductors, neglecting the coupling of the magnetic field to the orbital motion of electrons in the superconducting planes. For 
perpendicular field, measurements support their theory, strongly suggesting that $H_{c 2}$ is limited by the spin paramagnetism below a certain characteristic temperature $T^{*} \sim 0.85 T_{c}$. 24 For parallel field the role of the spin paramagnetism should be clarified with regard to the orbital effect.

Magnetization measurements GL-like Hao and Clem model.26 In higher fields, where the magnetic phase diagram contains a vortex fluid,20 one can presume that the diamagnetism gf the vortex fluid resembles closely to that of an ideal Abrikosov mixed state at temperatures not too close to $T_{c} .27$ In this regime, the measurements on cuprates should have the same slope in different fields, with strong temperature dependence due to the spin paramagnetism.

Electronic spin susceptibility measurements provide evidence about the pairing state.28 Recent ESR Knight shift measurements on YBCO of Jánossy et al.29 clearly show the temperature dependence characteristic for 2D d-wave superconductivity, different from the BCS theory prediction for 3D s-wave superconductors. 30

In Sec. II we derive the GL equations for cleap $2 \mathrm{D} d_{x^{2}-y^{2}}$ superconductors in the magnetic field parallel to the $c$

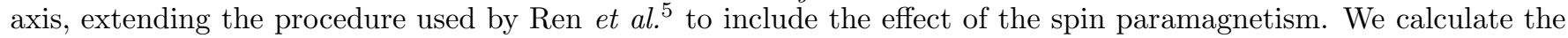
upper critical field and discuss the importance of the spin paramagnetism regarding the experimental data for YBCO in Sec. III. Within the model of layered superconductors as a stack of identical 2D conducting planes, we calculate the reversible magnetization in the Abrikosov approximation, Sec. IV, and the spin susceptibility, Sec. V. Section VI contains the conclusion.

\section{GINZBURG-LANDAU EQUATIONS}

Including the interaction of the electron spins with the magnetic field, the four coupled Gor'kov equations are

$$
\begin{gathered}
{\left[i \omega_{n}-\frac{1}{2 m}\left(-i \nabla+\frac{e}{c} \mathbf{A}\right)^{2}-\mu_{B} H+\mu\right] G_{\uparrow \uparrow}\left(\mathbf{x}, \mathbf{x}^{\prime}, \omega_{n}\right)-\int d \mathbf{x}^{\prime \prime} \Delta_{\uparrow \downarrow}\left(\mathbf{x}, \mathbf{x}^{\prime \prime}\right) F_{\downarrow \uparrow}^{+}\left(\mathbf{x}^{\prime \prime}, \mathbf{x}^{\prime}, \omega_{n}\right)=\delta\left(\mathbf{x}-\mathbf{x}^{\prime}\right),} \\
{\left[i \omega_{n}-\frac{1}{2 m}\left(-i \nabla+\frac{e}{c} \mathbf{A}\right)^{2}+\mu_{B} H+\mu\right] G_{\downarrow \downarrow}\left(\mathbf{x}, \mathbf{x}^{\prime}, \omega_{n}\right)-\int d \mathbf{x}^{\prime \prime} \Delta_{\downarrow \uparrow}\left(\mathbf{x}, \mathbf{x}^{\prime \prime}\right) F_{\uparrow \downarrow}^{+}\left(\mathbf{x}^{\prime \prime}, \mathbf{x}^{\prime}, \omega_{n}\right)=\delta\left(\mathbf{x}-\mathbf{x}^{\prime}\right),} \\
{\left[-i \omega_{n}-\frac{1}{2 m}\left(i \nabla+\frac{e}{c} \mathbf{A}\right)^{2}+\mu_{B} H+\mu\right] F_{\downarrow \uparrow}^{+}\left(\mathbf{x}, \mathbf{x}^{\prime}, \omega_{n}\right)+\int d \mathbf{x}^{\prime \prime} \Delta_{\downarrow \uparrow}^{+}\left(\mathbf{x}, \mathbf{x}^{\prime \prime}\right) G_{\uparrow \uparrow}\left(\mathbf{x}^{\prime \prime}, \mathbf{x}^{\prime}, \omega_{n}\right)=0,} \\
{\left[-i \omega_{n}-\frac{1}{2 m}\left(i \nabla+\frac{e}{c} \mathbf{A}\right)^{2}-\mu_{B} H+\mu\right] F_{\uparrow \downarrow}^{+}\left(\mathbf{x}, \mathbf{x}^{\prime}, \omega_{n}\right)+\int d \mathbf{x}^{\prime \prime} \Delta_{\uparrow \downarrow}^{+}\left(\mathbf{x}, \mathbf{x}^{\prime \prime}\right) G_{\downarrow \downarrow}\left(\mathbf{x}^{\prime \prime}, \mathbf{x}^{\prime}, \omega_{n}\right)=0 .}
\end{gathered}
$$

Here, $G_{\alpha \alpha}\left(\mathbf{x}, \mathbf{x}^{\prime}, \omega_{n}\right)$ and $F_{\alpha \beta}^{+}\left(\mathbf{x}, \mathbf{x}^{\prime}, \omega_{n}\right)$ are normal and anomalous Green's functions respectively $31 \omega_{n}=(2 n+1) \pi T$ are the Matsubara frequencies $\left(k_{B}=\hbar=1\right), \mu$ is the chemical potential and $\mu_{B}$ is the Bohr magneton. The magnetic field $\mathbf{H}=$ curlA is perpendicular to the superconducting plane. The order parameter $\Delta_{\uparrow \downarrow}^{+}$is defined by self-consistency equation

$$
\Delta_{\uparrow \downarrow}^{+}\left(\mathbf{x}, \mathbf{x}^{\prime \prime}\right)=V\left(\mathbf{x}-\mathbf{x}^{\prime \prime}\right)\left\langle\Psi_{\uparrow}^{\dagger}(\mathbf{x} t) \Psi_{\downarrow}^{\dagger}\left(\mathbf{x}^{\prime \prime} t\right)\right\rangle=-V\left(\mathbf{x}-\mathbf{x}^{\prime \prime}\right) T \sum_{\omega_{n}} F_{\uparrow \downarrow}^{+}\left(\mathbf{x}, \mathbf{x}^{\prime \prime}, \omega_{n}\right),
$$

where $V\left(\mathbf{x}-\mathbf{x}^{\prime \prime}\right)$ is the effective pairing interaction. $\Delta_{\downarrow \uparrow}^{+}\left(\mathbf{x}, \mathbf{x}^{\prime \prime}\right), \Delta_{\uparrow \downarrow}\left(\mathbf{x}, \mathbf{x}^{\prime \prime}\right)$ and $\Delta_{\downarrow \uparrow}\left(\mathbf{x}, \mathbf{x}^{\prime \prime}\right)$ are defined analogously.

From Eqs. 2.1 - (2.5), to the third order in $\Delta$, we find

$$
\begin{aligned}
& \Delta_{\uparrow \downarrow}^{+}(\mathbf{x}, \mathbf{y})=V(\mathbf{x}-\mathbf{y}) T \sum_{\omega_{n}}\left\{\int d \mathbf { x } ^ { \prime } d \mathbf { x } ^ { \prime \prime } \tilde { G } _ { \uparrow \uparrow } ( \mathbf { x } ^ { \prime } , \mathbf { x } , - \omega _ { n } ) \Delta _ { \uparrow \downarrow } ^ { + } ( \mathbf { x } ^ { \prime } , \mathbf { x } ^ { \prime \prime } ) \left[\tilde{G}_{\downarrow \downarrow}\left(\mathbf{x}^{\prime \prime}, \mathbf{y}, \omega_{n}\right)\right.\right. \\
& \left.\left.-\int d \mathbf{x}_{1} d \mathbf{x}_{2} \tilde{G}_{\downarrow \downarrow}\left(\mathbf{x}^{\prime \prime}, \mathbf{x}_{1}, \omega_{n}\right) \Delta_{\downarrow \uparrow}\left(\mathbf{x}_{1}, \mathbf{x}_{2}\right) \int d \mathbf{x}_{3} d \mathbf{x}_{4} \tilde{G}_{\uparrow \uparrow}\left(\mathbf{x}_{3}, \mathbf{x}_{2},-\omega_{n}\right) \Delta_{\uparrow \downarrow}^{+}\left(\mathbf{x}_{3}, \mathbf{x}_{4}\right) \tilde{G}_{\downarrow \downarrow}\left(\mathbf{x}_{4}, \mathbf{y}, \omega_{n}\right)\right]\right\} .
\end{aligned}
$$

Here $\tilde{G}_{\sigma}$ is the Green function in the normal state

$$
\tilde{G}_{\sigma}\left(\mathbf{x}, \mathbf{x}^{\prime}, \omega_{n}\right)=\sum_{\mathbf{k}} \frac{e^{i \mathbf{k}\left(\mathbf{x}-\mathbf{x}^{\prime}\right)}}{i \omega_{n}-\sigma \mu_{B} H-\epsilon_{\mathbf{k}}} e^{-i \frac{e}{c} \mathbf{A}(\mathbf{x}) \cdot\left(\mathbf{x}-\mathbf{x}^{\prime}\right)}
$$


where $\sigma= \pm 1$ corresponds to spin up and down. The classical approximation is used, taking account of the orbital effect by a change of phase, and $\epsilon_{\mathbf{k}}=\left(\mathbf{k}^{2} / 2 m\right)-\mu$.

In the notation of Ref. 5 , we find

$$
\begin{aligned}
& \Delta^{*}(\mathbf{R}, \mathbf{k})=\sum_{\mathbf{k}^{\prime}} V\left(\mathbf{k}-\mathbf{k}^{\prime}\right) T \sum_{\omega_{n}}\left[\frac{1}{\omega_{n}^{2}+\epsilon_{\mathbf{k}^{\prime}}^{2}}+\frac{\epsilon_{\mathbf{k}^{\prime}}^{2}-3 \omega_{n}^{2}}{\left(\omega_{n}^{2}+\epsilon_{\mathbf{k}^{\prime}}^{2}\right)^{3}} \mu_{B}^{2} H^{2}+\frac{\epsilon_{\mathbf{k}^{\prime}}^{2}-3 \omega_{n}^{2}}{\left(\omega_{n}^{2}+\epsilon_{\mathbf{k}^{\prime}}^{2}\right)^{3}} \frac{1}{4 m^{2}}\left(k_{x}^{\prime 2} \Pi_{x}^{2}+k_{y}^{\prime 2} \Pi_{y}^{2}\right)\right. \\
& \left.-\frac{1}{4 m} \frac{\epsilon_{\mathbf{k}^{\prime}}}{\left(\omega_{n}^{2}+\epsilon_{\mathbf{k}^{\prime}}^{2}\right)^{2}} \Pi^{2}\right] \Delta^{*}\left(\mathbf{R}, \mathbf{k}^{\prime}\right)-\sum_{\mathbf{k}^{\prime}} V\left(\mathbf{k}-\mathbf{k}^{\prime}\right) T \sum_{\omega_{n}} \frac{1}{\left(\omega_{n}^{2}+\epsilon_{\mathbf{k}^{\prime}}^{2}\right)^{2}}\left|\Delta^{*}\left(\mathbf{R}, \mathbf{k}^{\prime}\right)\right|^{2} \Delta^{*}\left(\mathbf{R}, \mathbf{k}^{\prime}\right),
\end{aligned}
$$

where $\Delta_{\downarrow \uparrow}^{+}=\Delta_{\uparrow \downarrow}^{+} \equiv \Delta^{*}, \mathbf{R}=(\mathbf{x}+\mathbf{y}) / 2$ is the center of mass coordinate, and $\boldsymbol{\Pi}=-i \partial / \partial \mathbf{R}-2 e \mathbf{A} / c$. The Fourier transform is performed with respect to the relative coordinate $\mathbf{r}=\mathbf{x}-\mathbf{y}$, and $(\pi T)^{2} \gg\left(\mu_{B} H\right)^{2}$ is assumed.12

For $d_{x^{2}-y^{2}}$ pairing, we take

$$
V\left(\mathbf{k}-\mathbf{k}^{\prime}\right)=V_{0}\left(\hat{k}_{x}^{2}-\hat{k}_{y}^{2}\right)\left(\hat{k}_{x}^{\prime 2}-\hat{k}_{y}^{\prime 2}\right)
$$

and

$$
\Delta^{*}(\mathbf{R}, \mathbf{k})=\Delta_{0}^{*}(\mathbf{R})\left(\hat{k}_{x}^{2}-\hat{k}_{y}^{2}\right)
$$

where $\hat{\mathbf{k}}$ is the unit vector in the direction of $\mathbf{k}$.

From Eq. (2.8), we obtain for $T$ near $T_{c}$ the first GL equation

$$
\Delta_{0}^{*}(\mathbf{R})=\frac{1}{2} N(0) V_{0} \Delta_{0}^{*} \ln \frac{2 C \omega_{c}}{\pi T}-\frac{7 \zeta(3)}{8} \frac{1}{\pi^{2} T_{c}^{2}} N(0) V_{0}\left[\frac{1}{8} v_{F}^{2} \Pi^{2} \Delta_{0}^{*}+\mu_{B}^{2} H^{2} \Delta_{0}^{*}+\frac{3}{8}\left|\Delta_{0}^{*}\right|^{2} \Delta_{0}^{*}\right],
$$

where $\omega_{c}$ is the energy cut-off, $C=1.78$ is the Euler constant, and 2D density of states for a given spin orientation $N(0)=m / 2 \pi$. This equation is the same as in Ref. 5 , with an additional term containing the Bohr magneton $\mu_{B}$.

In the same approach, from the quantum mechanical equation for a single electron of spin $\hat{\mathbf{s}} 22$

$$
\mathbf{j}=-\frac{i e}{2 m}\left[\left(\nabla \Psi^{*}\right) \Psi-\Psi^{*} \nabla \Psi\right]-\frac{e^{2}}{m c} \mathbf{A} \Psi^{*} \Psi-2 c \mu_{B} \operatorname{curl}\left(\Psi^{*} \hat{\mathbf{s}} \Psi\right),
$$

the superconducting current density is

$$
\begin{array}{r}
\mathbf{j}_{s}(\mathbf{x})=\frac{e T}{2 i m} \sum_{\omega_{n}} \sum_{\sigma} \int d \mathbf{x}_{1} d \mathbf{x}_{2} d \mathbf{x}_{3} d \mathbf{x}_{4} \Delta\left(\mathbf{x}_{1}, \mathbf{x}_{2}\right) \Delta^{*}\left(\mathbf{x}_{3}, \mathbf{x}_{4}\right) \tilde{G}_{-\sigma}\left(\mathbf{x}_{3}, \mathbf{x}_{2},-\omega_{n}\right) \\
\times\left.\left(\nabla_{\mathbf{x}}-\nabla_{\mathbf{y}}\right)\left[\tilde{G}_{\sigma}\left(\mathbf{x}, \mathbf{x}_{1}, \omega_{n}\right) \tilde{G}_{\sigma}\left(\mathbf{x}_{4}, \mathbf{y}, \omega_{n}\right)\right]\right|_{\mathbf{y}=\mathbf{x}} \\
+c T \mu_{B} \operatorname{curl} \sum_{\omega_{n}} \sum_{\sigma=-1}^{1} \vec{\sigma} \int d \mathbf{x}_{1} d \mathbf{x}_{2} d \mathbf{x}_{3} d \mathbf{x}_{4} \Delta\left(\mathbf{x}_{1}, \mathbf{x}_{2}\right) \Delta^{*}\left(\mathbf{x}_{3}, \mathbf{x}_{4}\right) \\
\times \tilde{G}_{\sigma}\left(\mathbf{x}, \mathbf{x}_{1}, \omega_{n}\right) \tilde{G}_{-\sigma}\left(\mathbf{x}_{3}, \mathbf{x}_{2},-\omega_{n}\right) \tilde{G}_{\sigma}\left(\mathbf{x}_{4}, \mathbf{x}, \omega_{n}\right),
\end{array}
$$

where $\vec{\sigma}$ is the unit vector orthogonal to the superconducting plane, $\vec{\sigma}=\sigma \mathbf{H} / H$. The first term in Eq. (2.13) is the diamagnetic current $\mathbf{j}_{s}^{d}$, and the second term is due to the difference between the paramagnetic current associated with the electron spins in the normal and the superconducting state, $\mathbf{j}_{s}^{\text {spin }}$. Note that the total current is $\mathbf{j}=$ $\mathbf{j}_{s}+2 N(0) \mu_{B}^{2}$ curlH, where the second term is the paramagnetic current in the normal state. Introducing the center of mass, performing the Fourier transform of $\Delta$ with respect to the relative coordinates, and after integrations similar as in Ref. 5, we get

$$
\begin{array}{r}
\mathbf{j}_{s}=\frac{e T}{m^{2}} \sum_{\omega_{n}} \int \frac{d \mathbf{k}}{(2 \pi)^{2}} \Delta^{*}(\mathbf{R}, \mathbf{k}) \frac{1}{\omega_{n}^{2}+\epsilon_{\mathbf{k}}^{2}}\left\{\left.\frac{m \mathbf{k}}{i \omega_{n}-\epsilon_{\mathbf{k}}}\left[\nabla_{\mathbf{k}^{\prime}} \cdot \mathbf{\Pi}^{*} \Delta\left(\mathbf{R}, \mathbf{k}^{\prime}\right)\right]\right|_{\mathbf{k}^{\prime}=-\mathbf{k}}\right. \\
\left.-\frac{2 \mathbf{k}}{\left(i \omega_{n}-\epsilon_{\mathbf{k}}\right)^{2}}\left[\mathbf{k} \cdot \mathbf{\Pi}^{*} \Delta(\mathbf{R},-\mathbf{k})\right]\right\}+ \text { H.c. } \\
+c T \mu_{B} \operatorname{curl} \sum_{\omega_{n}} \int \frac{d \mathbf{k}}{(2 \pi)^{2}} \Delta(\mathbf{R},-\mathbf{k}) \Delta^{*}(\mathbf{R}, \mathbf{k}) 2 \mu_{B} \mathbf{H} \frac{\epsilon_{\mathbf{k}}^{2}-3 \omega_{n}^{2}}{\left(\omega_{n}^{2}+\epsilon_{\mathbf{k}}^{2}\right)^{3}} .
\end{array}
$$


Here, in $\mathbf{j}_{s}^{d}$ the spin paramagnetic effect is neglected, as well as the orbital effect in $\mathbf{j}_{s}^{\text {spin }} 13$

For $d_{x^{2}-y^{2}}$ pairing, Eq. (2.10), after integratiop-pver $\mathbf{k}$, for $T$ near $T_{c}$, and assuming $(\pi T)^{2} \gg\left(\mu_{B} H\right)^{2}$, the second GL equation for the total supercurrent density is 33

$$
\mathbf{j}_{s}=\frac{7 \zeta(3) N(0) m v_{F}^{2}}{32 \pi^{2} T_{c}^{2}}\left[-\frac{i e}{m}\left(\Delta_{0} \frac{\partial \Delta_{0}^{*}}{\partial \mathbf{R}}-\Delta_{0}^{*} \frac{\partial \Delta_{0}}{\partial \mathbf{R}}\right)-\frac{4 e^{2}}{m c} \mathbf{A}\left|\Delta_{0}^{*}\right|^{2}-\frac{8 c}{m v_{F}^{2}} \mu_{B}^{2} \operatorname{curl}\left(\mathbf{H}\left|\Delta_{0}^{*}\right|^{2}\right)\right] .
$$

Finally, for a stack of identical 2D conducting planes in the magnetic field parallel to the $c$ axis, the corresponding GL equations in the standard form are

$$
\begin{gathered}
\alpha \psi+\beta|\psi|^{2} \psi+\frac{1}{2 m} \boldsymbol{\Pi}^{* 2} \psi+\eta H^{2} \psi=0, \\
\mathbf{j}_{s}=-\frac{i e}{m}\left(\psi \frac{\partial \psi^{*}}{\partial \mathbf{R}}-\psi^{*} \frac{\partial \psi}{\partial \mathbf{R}}\right)-\frac{4 e^{2}}{m c} \mathbf{A}|\psi|^{2}-2 c \eta \operatorname{curl}\left(\mathbf{H}|\psi|^{2}\right),
\end{gathered}
$$

where

$$
\begin{aligned}
& \alpha=\frac{16 \pi^{2} T_{c}^{2}}{7 \zeta(3) m v_{F}^{2}} \frac{T-T_{c}}{T_{c}}, \\
& \beta=\frac{48 \pi^{2} T_{c}^{2}}{7 \zeta(3) N^{*}(0) m^{2} v_{F}^{4}},
\end{aligned}
$$

and

$$
\eta=\frac{4}{m v_{F}^{2}} \mu_{B}^{2}
$$

Here $N^{*}(0)=N(0) / \delta, \delta$ being the average spacing between the planes. $B^{3}$

In this model, the GL equations for a d-wave, clean and layered superconductor are of the same form as in the isotropic and clean 3D s-wave case. However, besides the factor $2 / 3$ in parameters $\alpha, \beta, \eta$, the quantities $m, v_{F}, N(0)$ refer to the effective mass, Fermi velocity and density of states in $2 \mathrm{D}$ conducting $a b$ planes. Therefore, the coherence length is $\xi=\xi_{a b}=(1 / 2 m|\alpha|)^{1 / 2}$, the penetration depth $\lambda=\lambda_{a b}=\left(m c^{2} \beta / 16 \pi e^{2}|\alpha|\right)^{1 / 2}$, and the GL parameter

$$
\kappa=\frac{\sqrt{6} c T_{c}}{e v_{F}^{2}} \sqrt{\frac{\pi}{7 \zeta(3) N^{*}(0)}} .
$$

The free energy density corresponding to Eqs. (2.16) and (2.17) is

$$
F=F_{n}+\alpha|\psi|^{2}+\frac{\beta}{2}|\psi|^{4}+\frac{1}{2 m}\left|\boldsymbol{\Pi}^{*} \psi\right|^{2}+\frac{H^{2}}{8 \pi}+\eta H^{2}|\psi|^{2} .
$$

\section{UPPER CRITICAL FIELD}

Near the second order phase transition to the normal phase, from the linearized Eq. (2.16)

$$
\alpha \psi+\frac{1}{2 m} \boldsymbol{\Pi}^{* 2} \psi+\eta H^{2} \psi=0,
$$

we obtain for the upper critical magnetic field parallel to the $c$ axis

$$
H_{c 2}=\frac{e v_{F}^{2}}{8 c \mu_{B}^{2}}\left[\sqrt{1+\frac{256 \pi^{2} c^{2} \mu_{B}^{2} T_{c}^{2}}{7 \zeta(3) e^{2} v_{F}^{4}}\left(1-\frac{T}{T_{c}}\right)}-1\right] .
$$

The slope at $T_{c}$ is 


$$
\left.\frac{d H_{c 2}}{d T}\right|_{T_{c}}=-\frac{16 \pi^{2} c T_{c}}{7 \zeta(3) e v_{F}^{2}}
$$

and the GL expression without the Pauli paramagnetism correction is simply

$$
H_{c 2}^{0}=T_{c}\left|d H_{c 2} / d T\right|_{T c}\left(1-\frac{T}{T_{c}}\right) .
$$

We illustrate our results using the experimental data for YBCO.21 22 Samples are in the clean limit, and for fields above $1 \mathrm{~T}$ the spin-orbit scattering can be neglected.9 Taking the slope $\left.d H_{\mathrm{C}} d T\right|_{T c}=-1.9 \mathrm{~T} / \mathrm{K}$ from the magnetization measurements on optimally doped YBCO $\left(T_{c}=92 \mathrm{~K}\right)$ of Welp et al. 21 we find that at $T=0.7 T_{c}$ the spin pair breaking lowers the reritical field for 10\%, Fig. 1(a). However, it is evident that a larger slope corresponds

to the data of Nakagawa et al 22 We obtain with Eq. (3.2) a good fit of the experimental data for $T \gtrsim 0.5 T_{c}$, taking the slope $d H_{c 2} /\left.d T\right|_{T c}=-2.6 \mathrm{~T} / \mathrm{K}$ and $T_{c}=84.3 \mathrm{~K}$, Fig. 1(b). For this slope the paramagnetic correction is $-15 \%$ at $T=0.7 T_{c}$.

At low temperatures, where GL theory is not applicable, $H_{c 2}(T)$ becomes saturated. From the data of Nakagawa et al.,22 $H_{c 2}(0) \approx 110 \mathrm{~T}$. The Won and Maki expression $H_{c 2}(0)=-0.63 T_{c} d H_{c 2} /\left.d T\right|_{T c}$, relevant for $2 \mathrm{D}$ d-wave superconductors, for $T_{c}=84.3 \mathrm{~K}$ and the slope $-2.6 \mathrm{~T} / \mathrm{K}$ gives $H_{c 2}(0)=138 \mathrm{~T}$. Therefore, the spin paramagnetism, not taken into account in Ref. 7, but relevant in this case because the Clogston limit is 155 T, should lower $H_{c 2}(0)$ for $20 \%$. However, note that $H_{c 2}$ measurements strongly depend on experimental techniques 2324

The Fermi velocities corresponding to $d H_{c 2} /\left.d T\right|_{T c}=-1.9 \mathrm{~T} / \mathrm{K}, T_{c}=92 \mathrm{~K}$ and $d H_{c 2} /\left.d T\right|_{T c}=-2.6 \mathrm{~T} / \mathrm{K}, T_{c}=84.3$ $\mathrm{K}$ are $v_{F}=10.1 \cdot 10^{6} \mathrm{~cm} / \mathrm{s}$ and $v_{F}=8.3 \cdot 10^{6} \mathrm{~cm} / \mathrm{s}$, respectively. The latter value is closer to that obtained from independently measured Fermi energy and the effective mass, 3 giving $v_{F}=(7.6 \pm 0.9) \cdot 10^{6} \mathrm{~cm} / \mathrm{s}$.

\section{REVERSIBLE MAGNETIZATION}

The GL Eqs. (2.16), (2.17) in the dimensionless form are

$$
\left(i \frac{\nabla}{\kappa}-\mathbf{a}\right)^{2} f=f-|f|^{2} f-\gamma h^{2} f
$$

and

$$
-\operatorname{curlcurl} \mathbf{a}=|f|^{2} \mathbf{a}-\frac{i}{2 \kappa}\left(f^{*} \nabla f-f \nabla f^{*}\right)+\gamma \operatorname{curl}\left(\mathbf{h}|f|^{2}\right),
$$

where

$$
\gamma=\frac{32 \pi N^{*}(0) \mu_{B}^{2}}{3}\left(1-\frac{T}{T_{c}}\right)
$$

The dimensionless quantities are introduced with $\psi=\sqrt{-\alpha / \beta} f, \mathbf{R}=\lambda \tilde{\mathbf{R}}_{2} 2 e \xi \mathbf{A} / c=\mathbf{a}$, and $2 e \xi \lambda \mathbf{H} / c=\mathbf{h}$. 34 Eqs. (4.1)-(4.3) are of the same form as for isotropic 3D s-wave superconductor, 12 with different $\kappa$, and $\gamma$, Eqs. (2.21) and (4.3).

Following the Abrikosov approach 34 in the vicinity of the upper critical field parallel to the $c$ axis of the layered superconductor, we obtain

$$
\begin{gathered}
h=h_{e}-\frac{|f|^{2}}{2 \kappa^{\prime}}, \\
\overline{|f|^{2}}=\frac{1}{\kappa^{\prime}} \frac{h_{c 2}-h_{e}}{\left(1-1 / 2 \kappa^{\prime 2}\right) \beta}, \\
b=h_{e}-\left(h_{c 2}-h_{e}\right) \frac{1}{\left(2 \kappa^{\prime 2}-1\right) \beta} .
\end{gathered}
$$

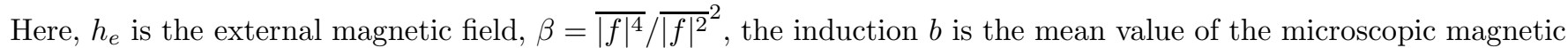
field $h$, and 


$$
\kappa^{\prime}=\frac{\kappa}{\sqrt{1+4 \kappa^{2} \gamma}}
$$

The dimensionless expression for the upper critical field, Eq. (3.2), is

$$
h_{c 2}=\frac{\sqrt{1+4 \kappa^{2} \gamma}-1}{2 \gamma \kappa} \text {. }
$$

We emphasize that in Eq. (4.6) magnetization due to the spin polarization of the normal electrons is not included. As in 3D s-wave case,12 the effect of the Pauli paramagnetism on $b$ is included simply by the scaling of $\kappa \rightarrow \kappa^{\prime}$. Therefore, the vortex lattice is again equilateral triangular lattice with $\beta=1.16$. The temperature dependence of $\kappa^{\prime}$ leads to the characteristic variation of the magnetization, $4 \pi m=b-h_{e}$, with temperature. From Eqs. (4.6)-(4.8), for $\kappa \gg 1$, the slope in physical units

$$
\frac{d M}{d H_{e}}=\frac{1+4 \kappa^{2} \gamma}{4 \pi \beta\left(2 \kappa^{2}-1\right)}=\left.\frac{d M}{d H_{e}}\right|_{T c}\left[1+\frac{256 \pi^{2} c^{2} \mu_{B}^{2} T_{c}^{2}}{7 \zeta(3) e^{2} v_{F}^{4}}\left(1-\frac{T}{T_{c}}\right)\right]
$$

is linearly decreasing with temperature, instead of the temperature independent slope in the GL approach without paramagnetic correction. Due to higher $T_{c}$ and smaller $v_{F}$, this effect is not negligible in high- $T_{c}$ cuprates, in contrast to the conventional superconductors. For $d H_{c 2} /\left.d T\right|_{T c}=-1.9 \mathrm{~T} / \mathrm{K}$, we obtain a strong variation $\left(d M / d H_{e}\right) /\left.\left(d M / d H_{e}\right)\right|_{T c}=1+1.4\left(1-T / T_{c}\right)$, or $1+2.6\left(1-T / T_{c}\right)$ for $d H_{c 2} /\left.d T\right|_{T c}=-2.6 \mathrm{~T} / \mathrm{K}$.

In high- $T_{c}$ superconductors the influence of the vortex lattice melting, and the strong fluctuation effects in the vicinity of $T_{c}$ also affect $d M / d H_{e}$ temperature dependence.20 However, because of the strong paramagnetic effect, the characteristic increase of $d M / d H_{e}$ with decreasing temperature, predicted by Eq. (4.9), should be dominant for $H \gtrsim 0.5 H_{c 2}$, and $0.5 \lesssim T / T_{c} \lesssim 0.9$. This effect may be compensatedonly by the strong temperature dependence of the effective parameter $\kappa_{2}$ within the extended GL theory approach. 35

\section{SPIN SUSCEPTIBILITY}

The current density due to the spin paramagnetism is

$$
\mathbf{j}_{s}^{\text {spin }}(\mathbf{r})=c \operatorname{curl}\left(\mathbf{M}_{s}^{\text {spin }}(\mathbf{r})-\mathbf{M}_{n}(\mathbf{r})\right)
$$

where $\mathbf{M}_{s}^{\operatorname{spin}}(\mathbf{r})$ and $\mathbf{M}_{n}(\mathbf{r})=2 N^{*}(0) \mu_{R}^{2} \mathbf{H}$ are the magnetization due to the spin polarization in the superconducting and in the normal state.13 From Eq. (5.1) and the last term in Eq. (2.17),

$$
M_{s}^{s p i n}=M_{n}-\frac{8 \mu_{B}^{2}}{m v_{F}^{2}} H|\psi|^{2} .
$$

In the weak magnetic field $|\psi|^{2}=-\alpha / \beta$, and the spin susceptibility $\chi_{s}=M_{s}^{\text {spin }} / H$ is

$$
\chi_{s}=\chi_{n}\left[1-\frac{4}{3}\left(1-\frac{T}{T_{c}}\right)\right] .
$$

This simple GL expression for the spin susceptibility of the layered d-wave superconductor is in a very good agreement with the microscopic theory result of Won and Maki, 1 in a large temperature range $T \gtrsim 0.6 T_{c}$. Analogously, the susceptibility in the GL approach for an isotropic 3D s-wave, as well as for 2D s-wave, superconductor is

$$
\chi_{s}=\chi_{n}\left[1-2\left(1-\frac{T}{T_{c}}\right)\right]
$$

in the agreement with the Yosida result from BCS theory. 30 Therefore, d-wave and s-wave symmetries of the order parameter lead to different slopes of the spin susceptibility curve in the high temperature regime.

This is illustrated in Fig. 2, in comparison with the ESR Knight shift data for YBCO of Jánossy et al.29 Small discrepancy of the experimental data and the theoretical predictions at high temperatures could be the consequence of the strong coupling effects 29 or of the presence of a small s-wave component of the order parameter which is possible in YBCO.d 


\section{CONCLUDING REMARKS}

The GL equations for layered d-wave clean superconductor are derived including the Pauli paramagnetism effect. The parallel upper critical field and the reversible magnetization in the Abrikosov approximation are calculated. The results are of the same form as obtained by Maki and Tsuneto in isotropic 3D s-wave case, with changed $\kappa$ and $\gamma$. The expression for $H_{c 2}$ gives an useful correction to the standard GL result, providing the correct determination of $d H_{c 2} /\left.d T\right|_{T c}$ for high- $T_{c}$ superconductors from the fit of experimental data in the relatively large range of temperatures below $T_{c}$. From the comparison with the experimental data of for $\mathrm{YBa}_{2} \mathrm{Cu}_{3} \mathrm{O}_{7-\delta}$ thin films 22 we obtain $d H_{c 2} /\left.d T\right|_{T c}=$ $-2.6 \mathrm{~T} / \mathrm{K}$, and conclude that the effect of Pauli paramagnetism on $H_{c 2}$ parallel to the $c$ axis is significant in comparison to the orbital effect, $-15 \%$ at $T=0.7 T_{c}$. At zero temperature, this effect should be greater, about $-20 \%$. The strong temperature dependence of the magnetization slope $d M / d H_{e}$ due to the spin paramagnetism influence, should be experimentally detectable in high field measurements. We have also derived the GL expression for the Knight shift, reflecting the pairing symmetry in accordance with the experimental data.

${ }^{1}$ M. Tinkham, Introduction to Superconductivity (McGraw Hill, New York, 1996), ch. 9.

${ }^{2}$ J. F. Annet, N. Goldenfeld, and A. J. Legget in: D. M. Ginsberg (Ed.), Physical Properties of High Temperature Superconductors, v. 5, (World Scientific, Singapure, 1996).

${ }^{3}$ D. R. Harshman and A. P. Mills, Jr., Phys. Rev. B 45, 10684 (1992).

${ }^{4}$ H. Won and K. Maki, Phys. Rev. B 49, 1397 (1994).

${ }^{5}$ Y. Ren, J. H. Xu, and C. S. Ting, Phys. Rev. Lett. 74, 3680 (1995).

${ }^{6}$ J. H. Xu, Y. Ren, and C. S. Ting, Phys. Rev. B 52, 7663 (1995).

${ }^{7}$ H. Won and K. Maki, Phys. Rev. B 53, 5927 (1996).

8 J. Shiraishi, M. Kohmoto, and K. Maki, Phys. Rev. B 59, 4497 (1999).

${ }^{9}$ K. Yang and S. L. Sondhi, Phys. Rev. B 57, 8566 (1998).

${ }^{10}$ H. Won, H. Jang, and K. Maki, cond-mat/9901252.

${ }^{11}$ L. P. Gor'kov, Zh. Eksp. Teor. Fiz. 36, 1918 (1959) [Sov. Phys. JETP 9, 1364 (1960)].

${ }^{12}$ K. Maki and T. Tsuneto, Progr. Theoret. Phys. 31, 945 (1964).

${ }^{13}$ K. Maki, Phys. Rev. 148, 362 (1966).

${ }^{14}$ A. P. Mackenzie, S. R. Julian, G. G. Lonzarich, A. Carrington, S. D. Hughes, R. S. Liu, and D. C. Sinclair, Phys. Rev. Lett. 71, 1238 (1993).

${ }^{15}$ M. S. Osofsky, R. J. Soulen, Jr., S. A. Wolf, J. M. Broto, H. Rakoto, J. C. Ousset, G. Coffe, S. Askenazy, P. Pari, I. Bozovic, J. N. Eckstein, and G. F. Virshup, Phys. Rev. Lett. 71, 2315 (1993).

${ }^{16}$ N. R. Werthamer, E. Helfand, and P. C. Hohenberg, Phys. Rev. 147, 295 (1966).

${ }^{17}$ Yu. N. Ovchinnikov and V. Z. Kresin, Phys. Rev. B 54, 1251 (1996); Europhys. Lett., 46, 794 (1999).

18 T. Koyama and M. Tachiki, Physica C 263, 25 (1996).

19 M. Rasolt and Z. Tešanović, Rev. Mod. Phys. 64, 709 (1992); S. Dukan and O. Vafek, Physica C 309,295 (1998).

${ }^{20}$ G. Blatter, M. V. Feigel'man, V. B. Geshkenbein, A. I. Larkin, and V. M. Vinokur, Rev. Mod. Phys. 66, 1125 (1994).

${ }^{21}$ U. Welp, W. K. Kwok, G. W. Crabtree, K. G. Vandervoort, and J. Z. Liu, Phys. Rev. Lett. 62, 1908 (1989).

${ }^{22}$ H. Nakagawa, T. Takamasu, N. Miura, and Y. Enomoto, Physica B 246-247, 429 (1998).

${ }^{23}$ H. Nakagawa, N. Miura, and Y. Enomoto, J. Phys.: Condens. Matter 10, 11571 (1998).

${ }^{24}$ J. L. O’Brien, H. Nakagawa, A. S. Dzurak, R. G. Clark, B. E. Kane, N. E. Lumpkin, N. Miura, E. E. Mitchell, J. D. Goettee, J. S. Brooks, D. G. Rickel, and R. P. Starrett, cond-mat/9901341.

${ }^{25}$ J. Sok, M. Xu, W. Chen, B. J. Suh, J. Gohng, D. K. Finnemore, M. J. Kramer, L. A. Schwartzkopf, and B. Dabrowski, Phys. Rev. B 51, 6035 (1995).

${ }^{26}$ Z. Hao and J. R. Clem, Phys. Rev. Lett. 67, 2371 (1991).

${ }^{27}$ Z. Hao, J. R. Clem, M. W. McElfresh, L. Civale, A. P. Malozemoff, and F. Holtzberg, Phys. Rev. B 43, 2844 (1991).

${ }^{28}$ D. Pines and P. Wróbel, Phys. Rev. B 53, 5915 (1996).

29 A. Jánossy, T. Fehér, G. Oszlányi, and G. V. M. Williams, Phys. Rev. Lett. 79, 2726 (1997).

${ }^{30}$ K. Yosida, Phys. Rev. 110, 769 (1958).

31 A. A. Abrikosov, L. P Gor'kov, and I. E Dzyaloshinski Methods of Quantum Field Theory in Statistical Physics (Dover, New York, 1975).

${ }^{32}$ L. D. Landau and E. M. Lifshitz, Quantum Mechanics (Addison-Wesley, Reading, 1958).

${ }^{33}$ Note that a factor 2 is missing in Eq. (17), Ref. 5, and in Eqs. (4.3)-(4.4), in Ref. 6.

${ }^{34}$ D. Saint-James, G. Sarma and E. J. Thomas, Type II Superconductivity (Pergamon Press, Oxford, 1969). 
${ }^{35}$ G. Eilenberger, Phys. Rev. 153, 584 (1967); K. Maki and T. Tsuzuki, Phys. Rev. 139, A868 (1965).

FIG. 1.

Temperature dependence of the parallel upper critical field of YBCO. Solid curve: GL result with the paramagnetic correction, Eq. (3.2). The weak coupling microscopic theory result without the paramagnetic correction (dotted curve), 0 and the corresponding GI result, Eq. (3.4), (dashedine) are shown for comparison. Solid circles represent $H_{c 2}$ measurements of Welp et al.21 (a), and Nakagawa et al.22 (b).

FIG. 2.

Temperature dependence of the normalized spin susceptibility. Solid lipes: GL approximation, Eqs. (5.3) and (5.4). Dotted curves: the weak coupling microscopic theory calculations 430 Top curves correspond to $2 \mathrm{D}$ d-wave, and bottom curves to 3D s-wave case. Solid circles: data of Jánossy et al.29 for YBCO. 


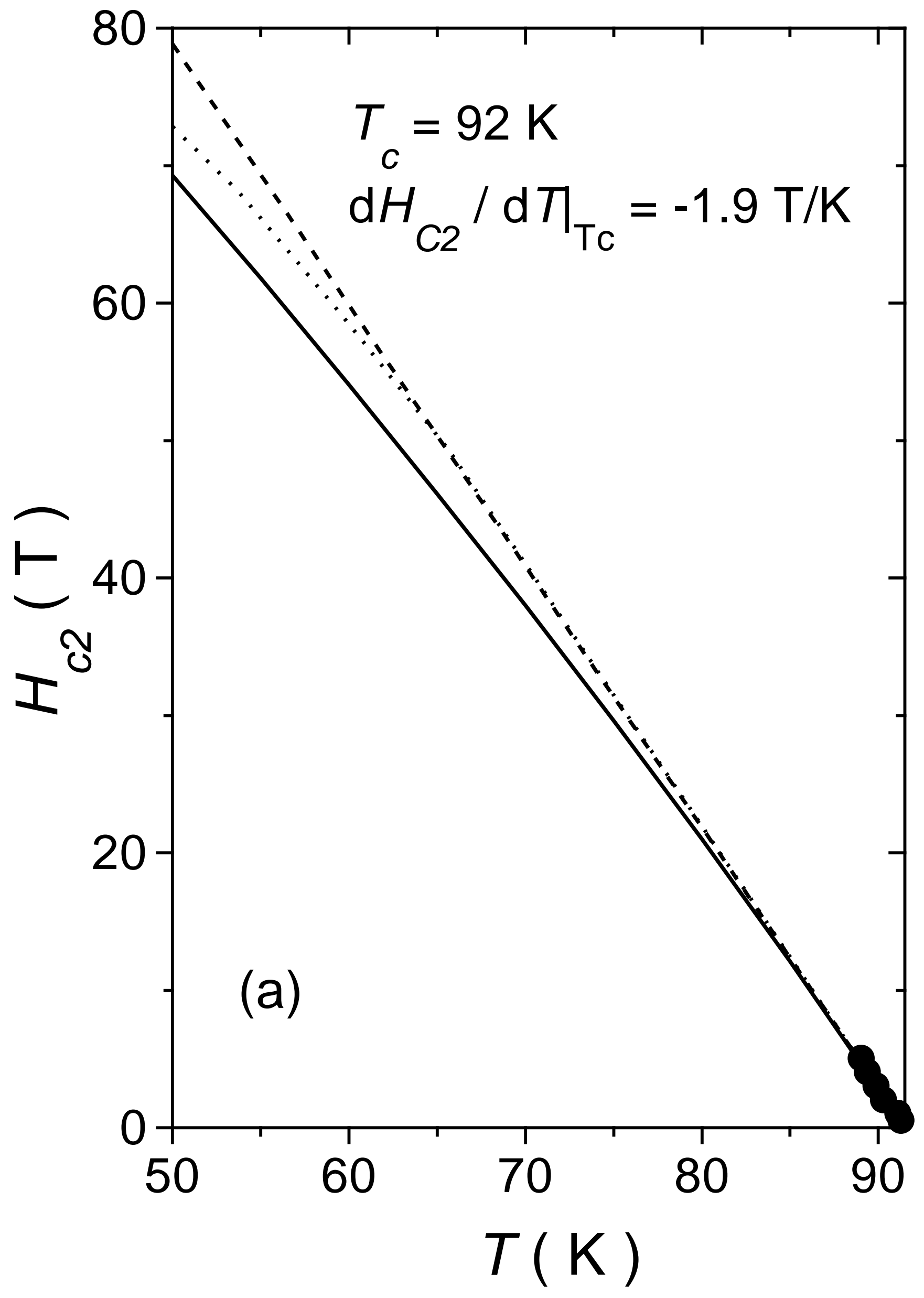




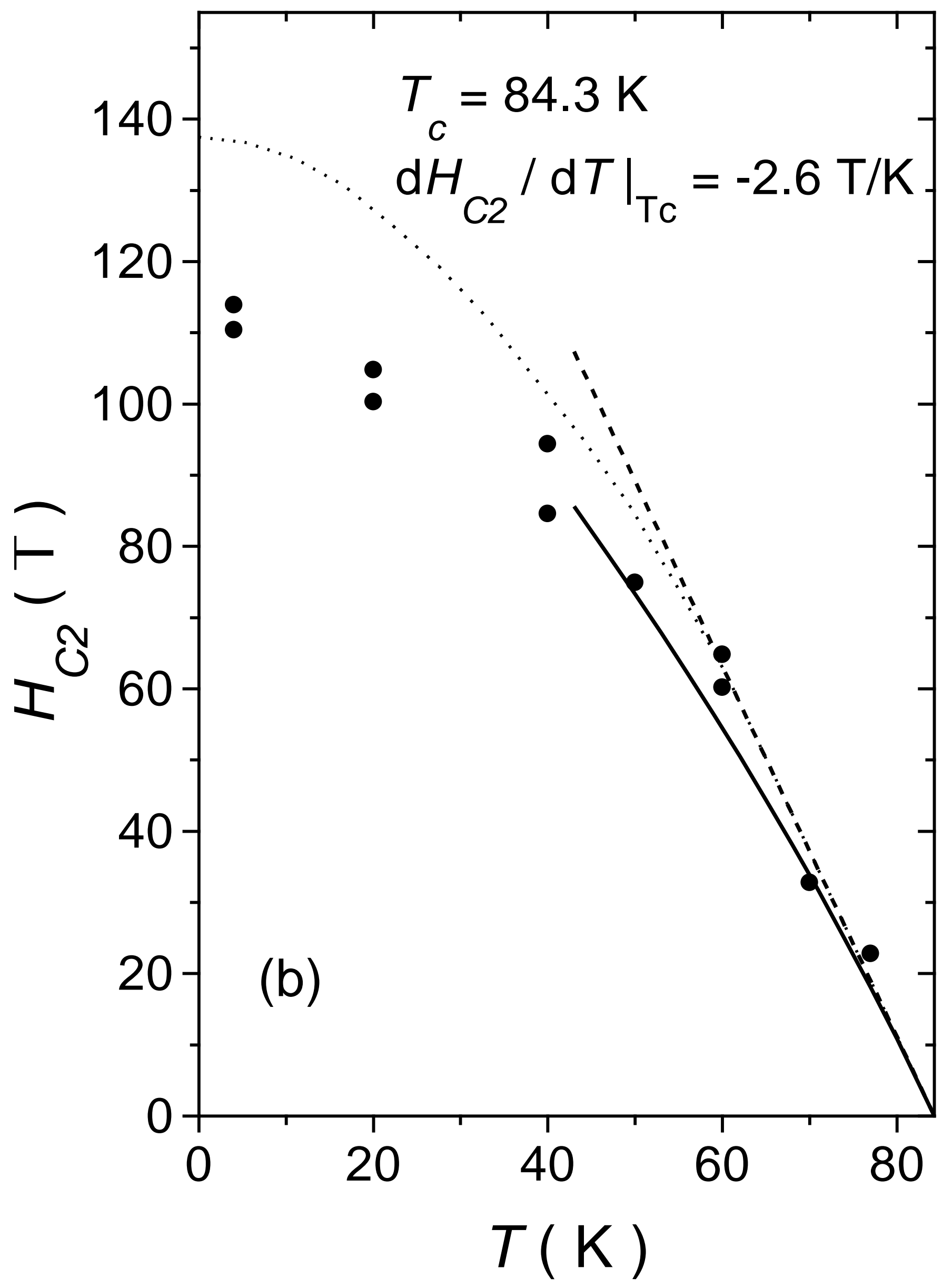




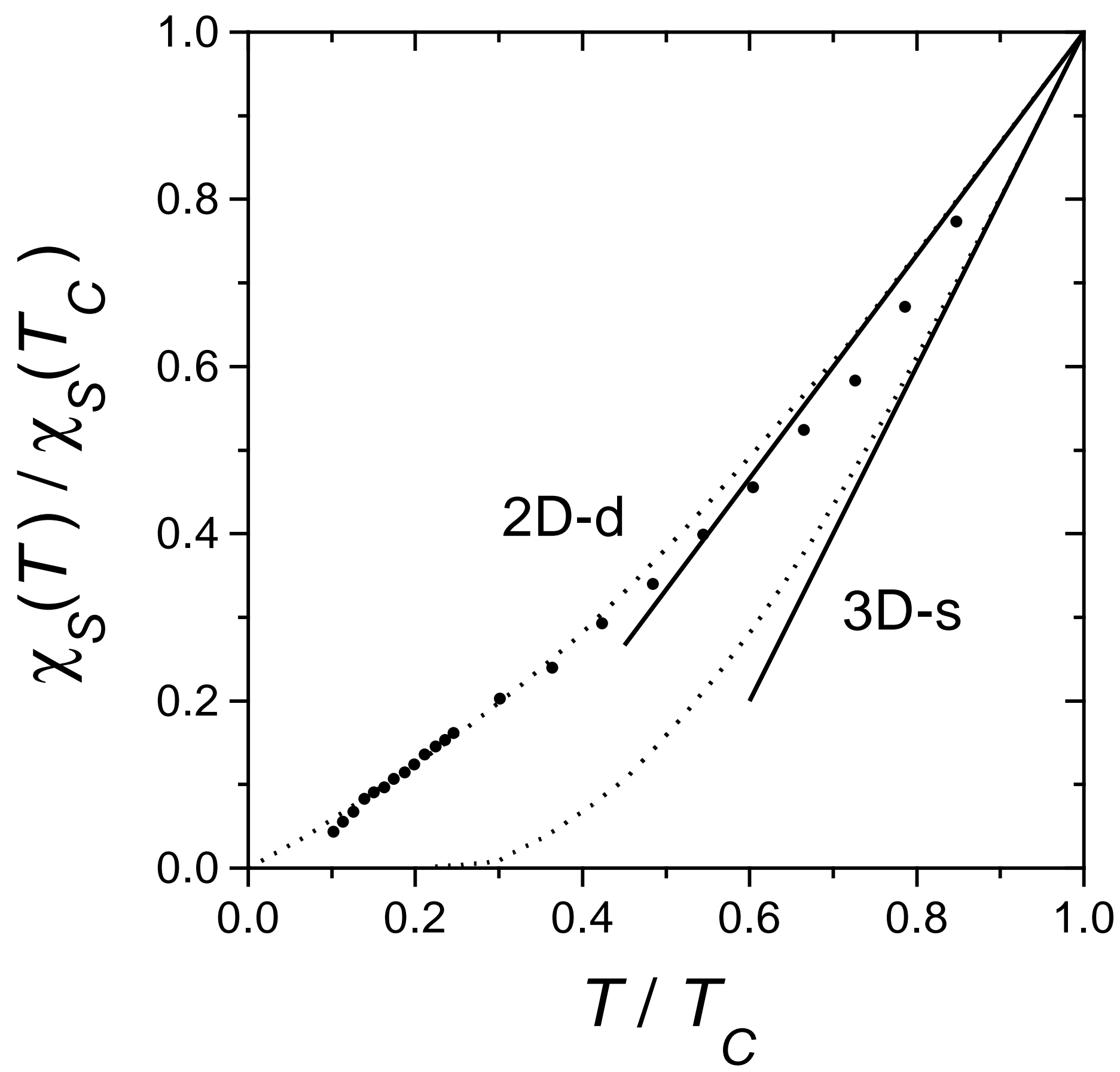

